\title{
Implementation of Policy Based Routing in MikroTik
}

\author{
${ }^{1}$ AYUB LAGHARI, ${ }^{1}$ NAFEESA BOHRA, AND \\ ${ }^{1}$ ABDUL LATIF MEMON \\ ${ }^{1}$ Institution of Information \& Communication Technologies, Mehran University of \\ Engineering \& Technology, Jamshoro, Pakistan. \\ ayub_laghari@ymail.com,nafeesa.zaki@faculty.muet.edu.pk, \\ abdul.latif@faculty.muet.edu.pk
}

\begin{abstract}
In this paper proper BW (Bandwidth) utilization is predicted using policy making decisions by using MikroTik router. Future network demands, high BW as number of devices are increasing day by day and to ensure this suitable network mechanism is needed with the help of policy making scenario. Implementing policies in firewall refrain the unauthorized access and $B W$ is not being efficiently utilized. The results suggest that MikroTik router provides built-in firewall that is not only user-friendly in policy making but also provide QoS (Quality of Service) and makes an efficient utilization of $B W$ resources. Policy based routing facilitates proper network management. Through policy application, network BW can be used appropriately, misuse of BW can be prevented which in turn can enhance QoS.
\end{abstract}

Key Words: $\quad$ MikroTik, Routing, Firewall, Bandwidth, Quality of Service.

\section{INTRODUCTION}

Since the origin of computer networking, networks control has remained central for its proper management and operation. Presently the system being used in businessbasic execution models or in any other field, to a great extent seems out of control. For effective network, each element in network types such as LAN (Local-Area Network), MAN (Metropolitan Area Network), WAN (Wide Area Network), VPNs (Virtual Private Network) are essential for proper service delivery. As use of BW is very important factor in current applications and services [1]. Now-a-days managing the network is quite complicated and much vulnerable in many organizations, such issues are unnoticed by many of the current clients. Intelligent and smart networks help in managing networks more efficiently and also are more reliable in throughput and E2E (End-to-End) service delivery [2]. Accessing the Internet has become vital these days. Be it for searching required information or for the sake of entertainment. For effective functioning of the Internet, the stable BW distribution by any ISP (Internet Service Provider) has become very crucial. For achieving this, a computer or 
device can be used to keep Internet speed under control. MikroTik is a device that facilitates BW management both in hardware as well as software. It also delivers the operating system that could be used to create a router. It is done by installing it into the computer. The tools provided in MikroTik Router are well-equipped to build a consistent and reliable router [3]. BW has been realized by ISPs as the most important part of a network. BW limiter is key in the creation of a fast and flexible access so that the Internet connection becomes stable according to the needs and thus BW management divides the amount of BW as needed. BW management is not hard, and therefore is proficiently utilized by any ISP for delivering the transfer speed to any client with a similar size or range without troubling the speed of another client [4]. In order to address the above issues, there is a need to formulate a mechanism that watches over traffic on the Internet network. MikroTik Router, is a software with built-in OS which makes computers a more reliable router. It covers characteristics comprising: firewall and NAT (Network Address Translation), directing, hotspot, P2P (Peer-to-Peer) tunneling protocol, DNS (Domain Name System) server, DHCP (Dynamic Host Configuration Protocol) server, hotspot and still a lot more features. MikroTik is designed as user-friendly and is utilized for the purposes of computer network administration for instance designing and constructing a simple too complicated computer network system. In networking, it is quite essential to ensure BW custom controlling consumed by every client's PC (Personal Computer). MikroTik routers offer multiple abilities that can execute BW distribution super vision for each client's computer. Therefore, by applying BW management, the network's QoS can be enhanced [5].

\section{RELATED WORK}

BW management plays a vital role in routers to enhance network management and performance management level and to unravel the approach they will go against or to solve the technological allegation at non-Wi-Fi Router regarding their purposeful limitations in DHCP Protocol. In a computer network where IP addresses are assigned manually the BW management may be worn out in a good way but IP (Internet Protocol) conflict arises once two computers have a similar IP. However, to avoid IP conflict once the network is in DHCP wherever IP is assigned during a random approach, BW for a specific IP address cannot be done properly [6].

It is significantly more conceivable to expect that the network fizzles since it just stays unrecognized. A system gives the reproducing ground to specialized advancement and yet it calls for an administration advancement without anyone else's input. To advance systems as a matter of first importance chiefs need to supplement the one of a kind rationale of systems. At exactly that point instruments and strategies can be placed enthusiastically that envision - and, best case scenario - anticipate disappointment of systems. It is believed the contentions are a motivation to reevaluate the administration of systems and to deliver the essential 
professionalization of the management - so that in future less systems come up short and more proceed onward effectively [7].

Adoption of a policy formation model for policy creation in an organization. The anticipated approach has been linked in BIUST (Botswana Universal College of Science and Innovation) network and confirmed its strength backed conferred results. Policy based networking is considered of impressive interest within research extreme and educational universities thanks to its benefits of delivering reliable, accurate and understandable network system [8].

Outcomes suggests when policy usage appeared that there's the necessity for approach execution in BIUST arrange framework as transmission capacity was moderated and utilized more proficiently as compared to results some time recently the approach was executed but there were still issues related to transmission capacity administration securing the information[9].

Strategy based administration frameworks accessible within the market place now-adays are more precisely portrayed as table-based frameworks. Much work is continuous at the IETF (Internet Engineering Task Force) for policy based systems, QoS, and security, however it will in general see the policy-based administration as an expansion of the directory-permitted systems with LDAP (Lightweight Directory Access Protocol) patterns [10]. It concentrates more on the network components as opposed to the total conduct of the system in general inside the setting of conveying an administration. In any case, the policy based methodology is a way to deal with the board that has extraordinary potential not just in overseeing basic administrations, such as the system component setup and VPNs, yet additionally in overseeing complex administrations. Much research is as yet expected to understand its maximum capacity [11-12].

\section{IMPLEMENTATION AND RESULTS}

MikroTik scenarios have been shown in Fig. 1. From Fig. 1, it can be seen that MikroTik router has capability of creating IP firewall and IP NAT that makes it very easy to give access to internal services, the most valuable feature is that it works on Application layer that can set the tone for the betterment of the network. Following are the steps for making a policy-based network:

Step-1: Basic LAN/WAN Connectivity

Basic LAN/WAN connectivity is done by assigning the following IP addresses:

Private IP: $\quad$ 100.127.1.2

Public IP: $\quad$ 192.168.3.3/24

Gateway: $\quad$ 100.127.1.1

DNS Server: 8.8.8.8

Login Name: admin 


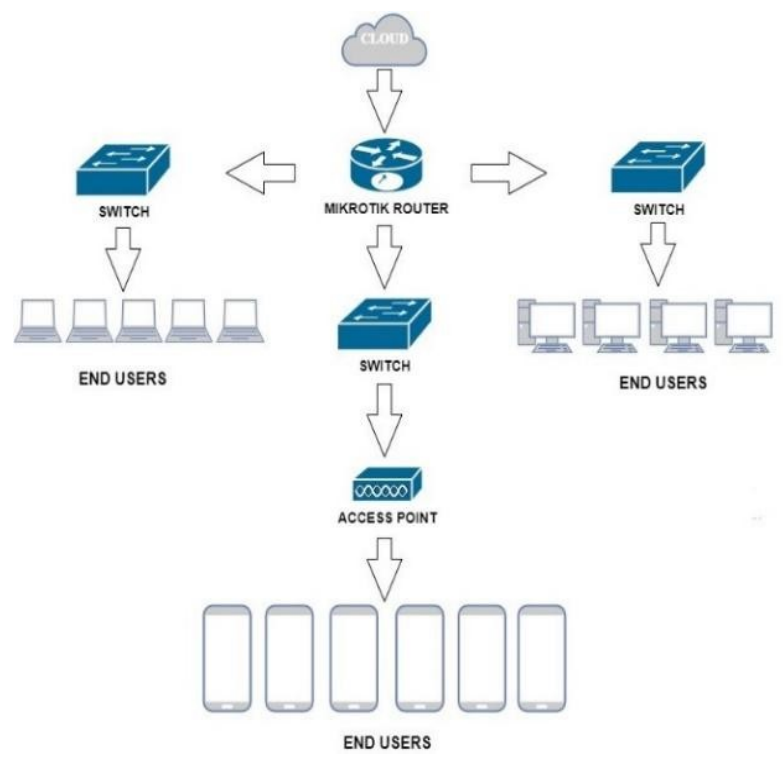

FIG. 1. MIKROTIK SCENARIO

\section{Step-2: Making a Route}

Routers utilized the data contained in a route to make choices about which network interface to forward a packet through in order to reach the destination address in the packet. Routers look up routes in the routing table to figure out how to move information from one system to another system. This means that one connection will use only one link in each direction, so ICMP (Internet Control Message Protocol) routes can be used to implement per-connection load balancing and is shown in Fig. 2. Gateway for DHCP setup-100.127.1.1Network will be identified with 100.127.1.254/24

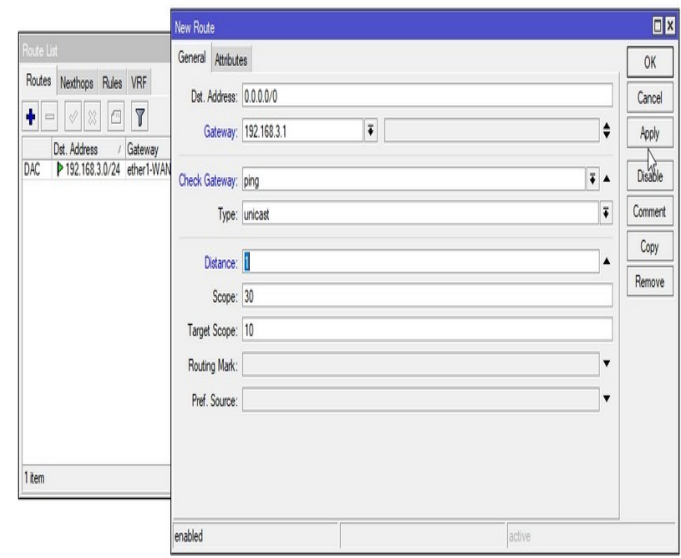

FIG. 2. ASSIGNING ROUTE 


\section{Step-3: Accessing Websites}

Fig. 3 shows that after the basic local area connectivity settings are configured, we can access the Internet. The next step is to configure NAT Rule. NAT is commonly referred to as NAT. A procedure to associate more than one computer to the web employing a single IP address. The sum of utilization of this strategy due to the constrained accessibility of IP addresses, the require for security, ease and adaptability in network management.

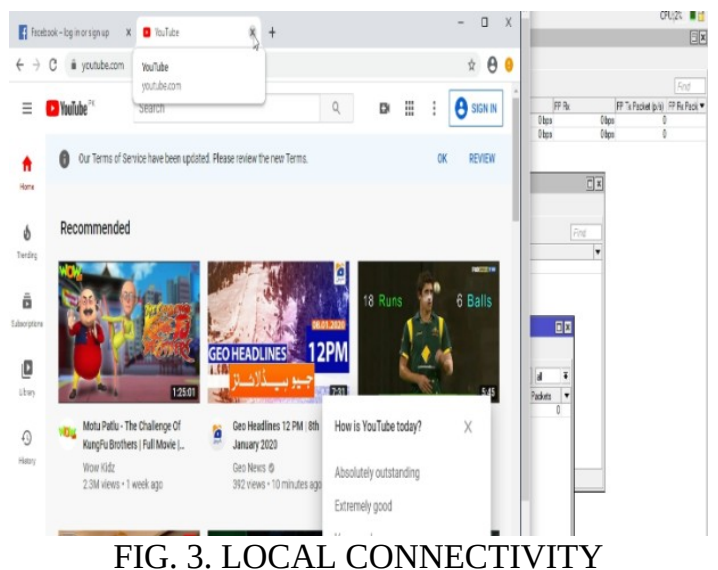

\section{Step-4: Firewall Filter Rule to Drop Packets}

Fig. 4shows after NAT Rule configuration is completed, the next step is to block social content to some of the users. This can be done by going to the menu. On the toolbar click firewall then in advanced tab we will add content that is to be blocked.

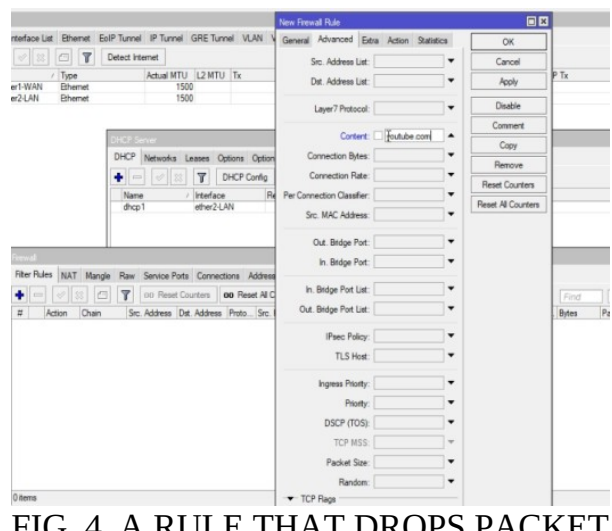

Fig. 5 shows that the rule we applied to block social websites has been implemented and it is working. To see the activities of each user can be done by using the Torch tool menuit serves for monitoring traffic in certain networks in real-time. 


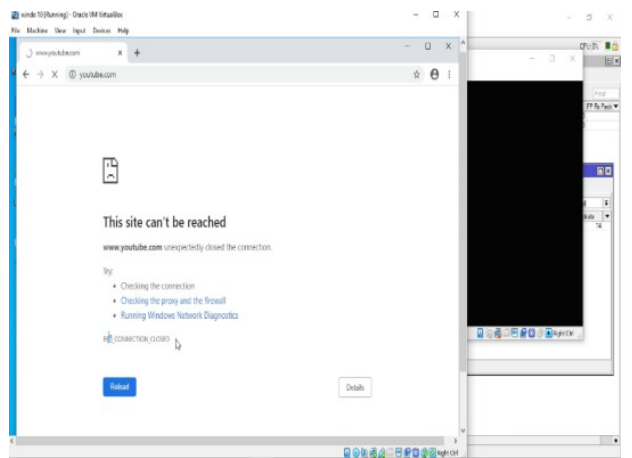

FIG. 5. CONTENT BLOCKED

\section{Step-5: Firewall Filter Rule to Access Social Websites}

Fig. 6 shows the firewall menu we will add the address in the address list that is given to the user to allow him the access to social websites.

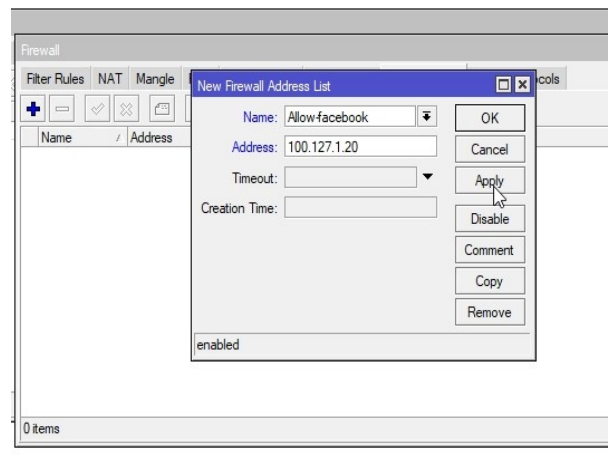

FIG. 6. FILTER RULE

\section{Step-6: DHCP Lease}

Fig. 7 shows the lease is characterized as the time period for which a DHCP server assigns a network address to a client. The lease might be prolonged (changed) upon consequent requests. If the client no longer requires the address, it can issue the address back to the server before the lease is up. This rule fixes IP in DHCP, user will get the same IP address that was addressed previously. 


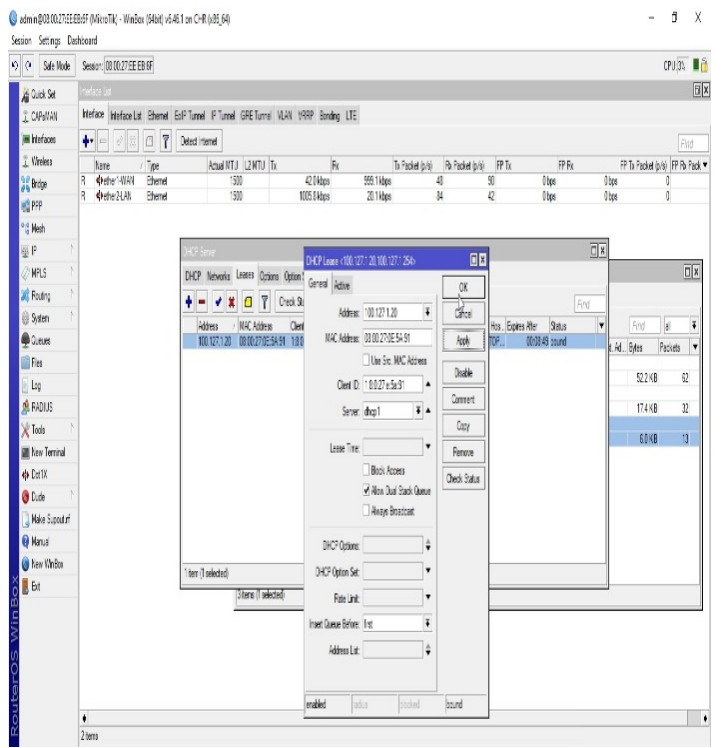

FIG. 7. FIXED IP - DHCP LEASE

\section{Step-7: Accessing Social Websites}

Fig. 8 shows that the user after requesting for accessing social websites can now access blocked content as the rule of IP fixed has been applied in firewall settings.

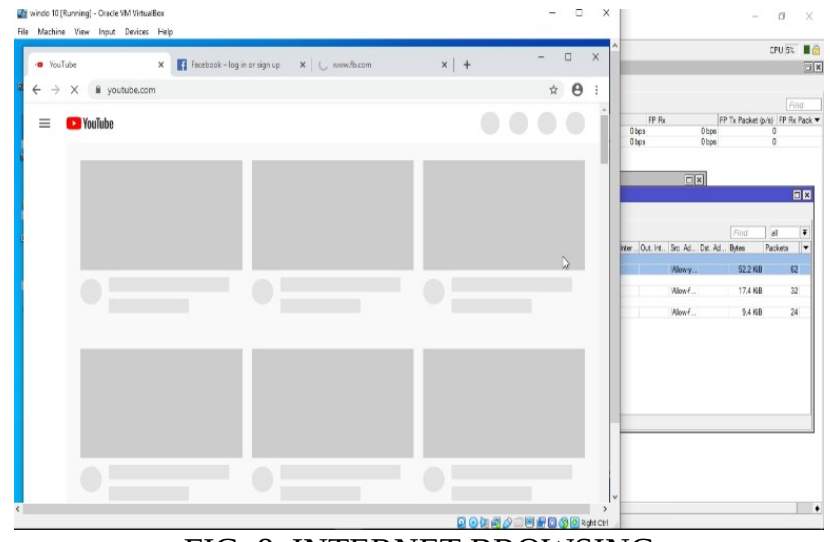

FIG. 8. INTERNET BROWSING

\section{CONCLUSION}

A policy-based network is important concern that must be seriously considered. In this paper, the concept of Policy Based Routing is discussed and implemented using MikroTik router. The policy-based strategy to organization has extraordinary potential not only in managing simple services such as the network element configuration and VPN, but also in handling complicated services such as a multilevel security service and an invasion management service. Results acquired after approach usage appeared that there is the requirement for strategy execution 
framework as data transfer capacity was rationed and utilized more effectively when contrasted with results before the strategy was actualized.

\section{ACKNOWLEDGEMENT}

Authors are thankful to Institution of Information \& Communication Technologies, Mehran University of Engineering \& Technology, Jamshoro, Pakistan, for providing facilities to carry out this research work.

\section{REFERENCES}

[1] Lubis, A., and Siahaan, A.P.U., "WLAN Penetration Examination of the University of Pembangunan Panca Budi”, International Journal of Engineering Trends and Technology, Volume 37, No. 3, pp. 165-168, 2016.

[2] Manesh T, Bhraguram, T.M., Rajaram, R., and Bhadran, V.K., "Network Forensic Investigation of HTTPS Protocol”, International Journal of Modern Engineering Research, Volume 3, No. 5, pp. 3096-3106, 2013.

[3] Ariyanto, H., andSiahaan, A.P.U., "Intrusion Detection System in Network Forensic Analysis and Investigation”, Journal of Computer Engineering, Volume 18, No. 6, pp. 115-121, 2016.

[4] Mollick, P., Biswas, S., Halder, A., and Salmani, M., "Mikrotik Router Configuration Using IPv6”, International Journal of Innovative Research in Computer, Volume 4, No. 2, pp. 2002007, 2016.

[5] Muhammad, D.L.S., Melva, P., and Siahaan, A.P.U., "MikroTik Bandwidth Management to Gain the Users Prosperity Prevalent”, International Journal of Emerging Trends \& Technology in Computer Science Volume 42. Pp. 218-222, [DOI: 10.14445/22315381/IJETTV42P243], 2016.

[6] Abdullah, I.M., "Bandwidth Management in Router for DHCP Protocol”, International Journal of Scientific and Engineering Research, Volume 10, pp. 1343-1346, [DOI: 10.14299/ijser.2019.03.03], 2019.

[7] Jules, T., and Christiane, F., "Failure of Networks and Network Management”, Strategies in Failure Management,[DOI: 10.1007/978-3-319-72757-8_14], June, 2018.

[8] Thato, S., Murtala, A., Rajalakshmi, S., Olefile, P., and Ontiretse, B., "Policy-Based Network Management in BIUST Network”, American Journal of Engineering \& Applied Sciences, Volume [DOI: 10.661-668. 10.3844/ajeassp.2017.661.668], 2017.

[9] Lymberopoulos, L., Lupu, E., and Sloman, M., “An Adaptive Policy-Based Framework for Network Services Management”, Journal of Network and Systems Management, Volume 11, No. 277, 2003.

[10] Choudhary, A.R., "Service Intelligence through Agile Information Controls”, Bell Labs Technical Journal, Volume 8, No. 4, pp. 61-70, 2003.

[11] Choudhary, A.R., "Policy-Based Network Management”, Bell Labs Technical Journal, Volume 9, pp. 19-29, [DOI: 10.1002/bltj.20002], 2004.

[12] Strassner, J., Moore, B., Moats, R., and Ellesson, E., "Policy Core LDAP Schema”, Internet Engineering Task Force, Internet Draft, October, 2002. 